\title{
Front Matter Volume 8623
}

, "Front Matter Volume 8623," Proc. SPIE 8623, Ultrafast Phenomena and Nanophotonics XVII, 862301 (21 March 2013); doi: 10.1117/12.2022469 


\title{
PROCEEDINGS OF SPIE
}

\section{Ultrafast Phenomena and Nanophotonics XVII}

\author{
Markus Betz \\ Abdulhakem Y. Elezzabi \\ Jin-Joo Song \\ Kong-Thon Tsen \\ Editors
}

3-6 February 2013

San Francisco, California, United States

Sponsored by

SPIE

Cosponsored by

Femtolasers, Inc. (United States)

Published by

SPIE 
The papers included in this volume were part of the technical conference cited on the cover and title page. Papers were selected and subject to review by the editors and conference program committee. Some conference presentations may not be available for publication. The papers published in these proceedings reflect the work and thoughts of the authors and are published herein as submitted. The publisher is not responsible for the validity of the information or for any outcomes resulting from reliance thereon.

Please use the following format to cite material from this book:

Author(s), "Title of Paper," in Ultrafast Phenomena and Nanophotonics XVII, edited by Markus Betz, Abdulhakem Y. Elezzabi, Jin-Joo Song, Kong-Thon Tsen, Proceedings of SPIE Vol. 8623 (SPIE, Bellingham, WA, 2013) Article CID Number.

ISSN: 0277-786X

ISBN: 9780819493927

Published by

SPIE

P.O. Box 10, Bellingham, Washington 98227-0010 USA

Telephone +1 3606763290 (Pacific Time) · Fax +1 3606471445

SPIE.org

Copyright (@ 2013, Society of Photo-Optical Instrumentation Engineers.

Copying of material in this book for internal or personal use, or for the internal or personal use of specific clients, beyond the fair use provisions granted by the U.S. Copyright Law is authorized by SPIE subject to payment of copying fees. The Transactional Reporting Service base fee for this volume is $\$ 18.00$ per article (or portion thereof), which should be paid directly to the Copyright Clearance Center (CCC), 222 Rosewood Drive, Danvers, MA 01923. Payment may also be made electronically through CCC Online at copyright.com. Other copying for republication, resale, advertising or promotion, or any form of systematic or multiple reproduction of any material in this book is prohibited except with permission in writing from the publisher. The CCC fee code is 0277-786X/13/\$18.00.

Printed in the United States of America.

Publication of record for individual papers is online in the SPIE Digital Library.

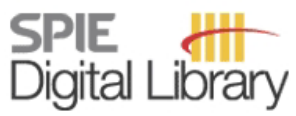

SPIEDigitalLibrary.org

Paper Numbering: Proceedings of SPIE follow an e-First publication model, with papers published first online and then in print and on CD-ROM. Papers are published as they are submitted and meet publication criteria. A unique, consistent, permanent citation identifier (CID) number is assigned to each article at the time of the first publication. Utilization of CIDs allows articles to be fully citable as soon as they are published online, and connects the same identifier to all online, print, and electronic versions of the publication. SPIE uses a six-digit CID article numbering system in which:

- The first four digits correspond to the SPIE volume number.

- The last two digits indicate publication order within the volume using a Base 36 numbering

system employing both numerals and letters. These two-number sets start with 00, 01, 02, 03, 04, $05,06,07,08,09,0 A, 0 B \ldots 0 Z$, followed by 10-1Z, 20-2Z, etc.

The CID Number appears on each page of the manuscript. The complete citation is used on the first page, and an abbreviated version on subsequent pages. Numbers in the index correspond to the last two digits of the six-digit CID Number. 


\title{
Contents
}

\author{
ix Conference Committee \\ xi Group IV photonics for the mid infrared (Plenary Paper) [8629-1] \\ $R$. Soref, The Univ. of Massachusetts at Boston (United States) \\ xxvii Light in a twist: optical angular momentum (Plenary Paper) [8637-2] \\ M. J. Padgett, Univ. of Glasgow (United Kingdom)
}

\section{THZ SPECTROSCOPY OF WATER AND BIOLOGICAL MOLECULES I}

862302 THz Raman spectra of aqueous solutions of hydrophiles and amphiphiles (Invited Paper) [8623-1]

I. A. Heisler, K. Mazur, S. R. Meech, The Univ. of East Anglia Norwich (United Kingdom)

862303 Ultrabroadband terahertz spectroscopies of biomolecules and water (Invited Paper)

[8623-2]

D. Turton, Univ. of Glasgow (United Kingdom); T. Harwood, Univ. of Strathclyde (United

Kingdom); A. Lapthorn, Univ. of Glasgow (United Kingdom); E. Ellis, Univ. of Strathclyde

(United Kingdom); K. Wynne, Univ. of Glasgow (United Kingdom)

\section{THZ SPECTROSCOPY OF WATER AND BIOLOGICAL MOLECULES II}

862305 Measuring phonons in protein crystals (Invited Paper) [8623-4]

G. Acbas, K. A. Niessen, D. K. George, Univ. at Buffalo, SUNY (United States); E. Snell, Univ. at Buffalo, SUNY (United States) and Hauptman Woodward Medical Research Institute (United States); A. G. Markelz, Univ. at Buffalo, SUNY (United States)

862306 Low-frequency dynamics of proteins and aqueous solutions studied by terahertz timedomain spectroscopy (Invited Paper) [8623-5]

N. Yamamoto, A. Ishihara, A. Kaneko, H. Iguchi, O. Kambara, A. Tamura, K. Tominaga, Kobe Univ. (Japan)

\section{COHERENT SPIN DYNAMICS}

862307 Ultrafast terahertz spin dynamics: from phonon-induced spin order to coherent magnon control (Invited Paper) [8623-6]

K. W. Kim, Univ. Konstanz (Germany) and Chungbuk National Univ. (Korea, Republic of); M. Porer, Univ. Konstanz (Germany) and Univ. Regensburg (Germany); C. Bernhard, Univ. de Fribourg (Switzerland); J. Demsar, A. Pashkin, Univ. Konstanz (Germany); T. Kampfrath, Fritz-Haber-Institut der Max-Planck-Gesellschaft (Germany); A. Sell, Univ. Konstanz (Germany); M. Wolf, Chungbuk National Univ. (Korea, Republic of); A. Leitenstorfer, Univ. Konstanz (Germany); R. Huber, Univ. Konstanz (Germany) and Univ. Regensburg (Germany) 
862308 Ultrafast mid-infrared spectroscopy of the charge- and spin-ordered nickelates (Invited Paper) [8623-7]

G. Coslovich, B. Huber, Lawrence Berkeley National Lab. (United States); W.-S. Lee, SLAC National Accelerator Lab. (United States); Y.-D. Chuang, Y. Zhu, Lawrence Berkeley National Lab. (United States); T. Sasagawa, Tokyo Institute of Technology (Japan); Z. Hussain, H. A. Bechtel, M. C. Martin, R. W. Schoenlein, Lawrence Berkeley National Lab. (United States); Z.-X. Shen, SLAC National Accelerator Lab. (United States); R. A. Kaindl, Lawrence Berkeley National Lab. (United States)

8623 OA Spin relaxation in spin light-emitting diodes: effects of magnetic field and temperature (Best Student Paper) [8623-9]

H. Höpfner, C. Fritsche, A. Ludwig, A. Ludwig, Ruhr-Univ. Bochum (Germany); F. Stromberg, H. Wende, W. Keune, Univ. Duisburg-Essen (Germany); D. Reuter, Univ. Paderborn (Germany); A. D. Wieck, N. C. Gerhardt, M. R. Hofmann, Ruhr-Univ. Bochum (Germany)

8623 OB Electron spin relaxation dynamics in GaN: influence of temperature, doping density, and crystal orientation [8623-10]

J. H. Buß, J. Rudolph, S. Starosielec, A. Schaefer, Ruhr-Univ. Bochum (Germany); F. Semond, Ctr. de Recherche sur l'Hétéro-Epitaxie et ses Applications, CNRS (France); D. Hägele, RuhrUniv. Bochum (Germany)

ULTRAFAST PHENOMENA IN SEMICONDUCTORS AND INSULATORS

8623 OD Ultrafast dynamics in topological insulators (Invited Paper) [8623-12]

C. W. Luo, H.-J. Chen, H. J. Wang, S. A. Ku, K. H. Wu, T. M. Uen, J. Y. Juang, J.-Y Lin, B. L. Young, T. Kobayashi, National Chiao Tung Univ. (Taiwan); R. Sankar, F. C. Chou, National Taiwan Univ. (Taiwan); H. Berger, Ecole Polytechnique Fédérale de Lausanne (Switzerland); G. D. Gu, Brookhaven National Lab. (United States)

8623 OE Two-photon physics with quantum-dot biexcitons (Invited Paper) [8623-13]

S. Schumacher, A. Zrenner, Univ. Paderborn (Germany)

NONLINEAR METAMATERIALS

$8623 \mathrm{OL}$ Optimal second-harmonic generation in split-ring resonator arrays [8623-20]

Y. Grynko, T. Meier, Univ. Paderborn (Germany); S. Linden, Karlsruher Institut für Technologie (Germany) and Rheinische Friedrich-Wilhelms-Univ. Bonn (Germany); F. B. P. Niesler, M. Wegener, Karlsruher Institut für Technologie (Germany); J. Förstner, Univ. Paderborn (Germany)

\section{NONLINEAR THZ INTERACTION AND SPECTROSCOPY}

8623 ON Terahertz semiconductor nonlinear optics (Invited Paper) [8623-22]

D. Turchinovich, Max-Planck-Institut für Polymerforschung (Germany) and DTU Fotonik, Technische Univ. Denmark (Denmark); J. M. Hvam, DTU Fotonik, Technische Univ. Denmark (Denmark); M. C. Hoffmann, SLAC National Accelerator Lab. (United States) 
862300 Observation of strong and broadband terahertz induced electroabsorption in multiple quantum wells [8623-23]

C.-Y. Li, The Univ. of New Mexico (United States); D. V. Seletskiy, The Univ. of New Mexico (United States) and Univ. Konstanz (Germany); J. G. Cederberg, Sandia National Labs. (United States); M. Sheik-Bahae, The Univ. of New Mexico (United States)

ULTRAFAST PROCESSES IN GRAPHENE AND CARBON NANOTUBES I

8623 OS Interaction of single-layer CVD graphene with a metasurface of terahertz split-ring resonators [8623-31]

F. Valmorra, G. Scalari, C. Maissen, ETH Zurich (Switzerland); W. Fu, C. Schönenberger, Univ. Basel (Switzerland); J. W. Choi, H. G. Park, M. Beck, J. Faist, ETH Zurich (Switzerland)

\section{ULTRAFAST NANOPLASMONICS}

8623 oW Purcell factor of metallic nanoresonators [8623-28]

C. Sauvan, Lab. Photonique, Numérique et Nanosciences, CNRS, Univ. Bordeaux 1 (France); J.-P. Hugonin, I. S. Maksymov, Lab. Charles Fabry, CNRS, Univ. Paris-Sud (France); P. Lalanne, Lab. Photonique, Numérique et Nanosciences, CNRS, Univ. Bordeaux 1 (France)

\section{STRONG-FIELD AND ATTOSECOND PHENOMENA}

862311 Photoemission at metallic nanostructures: multiphoton and strong-field dynamics (Invited Paper) [8623-36]

G. Herink, D. R. Solli, L. Wimmer, M. Gulde, K. Echternkamp, S. V. Yalunin, R. Bormann, C. Ropers, Univ. Göttingen (Germany)

862312 Probing ultrafast electron dynamics in condensed matter with attosecond photoemission (Invited Paper) [8623-37]

S. Neppl, Max-Planck-Institut für Quantenoptik (Germany); R. Ernstorfer, Fritz-Haber-Institut der Max-Planck-Gesellschaft (Germany); A. L. Cavalieri, Univ. of Hamburg (Germany); J. V. Barth, D. Menzel, Technische Univ. München (Germany); F. Krausz, Max-Planck-Institut für Quantenoptik (Germany); P. Feulner, Technische Univ. München (Germany); R. Kienberger, Max-Planck-Institut für Quantenoptik (Germany)

862313 Attosecond physics at a nanoscale metal tip: strong field physics meets near-field optics (Invited Paper) [8623-38]

M. Krüger, S. Thomas, M. Förster, L. Maisenbacher, Max-Planck-Institut für Quantenoptik (Germany); G. Wachter, C. Lemell, J. Burgdörfer, Technische Univ. Wien (Austria); P. Hommelhoff, Max-Planck-Institut für Quantenoptik (Germany) and Friedrich-AlexanderUniv. Erlangen-Nürnberg (Germany) 
862317 Efficient generation of record-short and record-long wavelengths based on backward and forward parametric interaction in lithium niobate (Invited Paper) [8623-42]

R. Chen, G. Sun, X. Lin, Y. J. Ding, Lehigh Univ. (United States)

862319 Laser pulse propagation in relativistically time-dependent media (Invited Paper) [8623-44] D. Faccio, Heriot-Watt Univ. (United Kingdom); E. Rubino, Univ. degli Studi dell'Insubria (Italy); A. Couairon, Ctr. de Physique Théorique, CNRS, Ecole Polytechnique (France); F. Belgiorno, Politecnico di Milano (Italy); F. Dalla Piazza, S. L. Cacciatori, Univ. degli Studi dell'Insubria (Italy)

8623 IA Simultaneous generation and coherent control of terahertz and XUV using two-color laser field [8623-45]

A. Gragossian, The Univ. of New Mexico (United States); D. V. Seletskiy, The Univ. of New Mexico (United States) and Univ. Konstanz (Germany); M. Sheik-Bahae, The Univ. of New Mexico (United States)

8623 1B Accessing new types of photocurrents using polarization-shaped excitation pulses [8623-46]

S. Priyadarshi, K. Pierz, M. Bieler, Physikalisch-Technische Bundesanstalt (Germany)

ULTRAFAST PROCESSES IN GRAPHENE AND CARBON NANOTUBES II

$8623 \mathrm{IF}$ Dynamics of excitons and trions in semiconducting carbon nanotubes (Invited Paper) [8623-50]

M. Okano, T. Nishihara, Y. Yamada, Y. Kanemitsu, Kyoto Univ. (Japan)

ULTRAFAST NANOPHOTONICS

$86231 \mathrm{~J}$ Supported plasmonic nanocrystals improve the performance of novel fiber-based sensors (Invited Paper) [8623-54]

A. Ianoul, J. Albert, Carleton Univ. (Canada)

$8623 \mathrm{lL} \quad$ Ultrafast adiabatic control of reciprocity and coherent backscattering in random scattering media [8623-56]

O. L. Muskens, Univ. of Southampton (United Kingdom); T. van der Beek, FOM Institute AMOLF (Netherlands); T. Wellens, Albert-Ludwigs-Univ. Freiburg (Germany)

\section{PLASMONICS AND METAMATERIALS}

$86231 \mathrm{M}$

Influence of resonator design on ultrastrong coupling between a two-dimensional electron gas and a THz metamaterial [8623-57]

C. Maissen, G. Scalari, F. Valmorra, C. Reichl, ETH Zurich (Switzerland); D. Schuh, Univ. Regensburg (Germany); W. Wegscheider, M. Beck, ETH Zurich (Switzerland); S. de Liberato, D. Hagenmüller, C. Ciuti, Lab. Matériaux et Phénomènes Quantiques, Univ. Paris 7-Denis Diderot, CNRS (France); J. Faist, ETH Zurich (Switzerland) 
8623 iN InGaAs amplifier for loss-compensation in nanoplasmonic circuits [8623-58]

M. Nielsen, A. Y. Elezzabi, Univ. of Alberta (Canada)

8623 IP Measurements of giant second harmonic generation from vertically aligned silicon nanowires [8623-62]

M. A. Swillam, The American Univ. in Cairo (Egypt); M. Khorasaninjead, S. S. Sainai, Univ. of Waterloo (Canada)

8623 IR Plasmonic slot waveguides with core nonlinearity [8623-60]

S. A. Tawfik, M. A. Swillam, The American Univ. in Cairo (Egypt)

POSTER SESSION

8623 is Wavelength tunable $\mathrm{GHz}$ repetition rate picosecond pulse generator using an SBS frequency comb [8623-63]

V. Lambin lezzi, S. Loranger, R. Kashyap, Ecole Polytechnique de Montréal (Canada)

8623 IV Air-breakdown coherent detection of terahertz using controlled optical bias [8623-66] C.-Y. Li, The Univ. of New Mexico (United States); D. V. Seletskiy, The Univ. of New Mexico (United States) and Univ. Konstanz (Germany); M. Sheik-Bahae, The Univ. of New Mexico (United States)

Author Index 


\section{Conference Committee}

Symposium Chair

David L. Andrews, University of East Anglia Norwich (United Kingdom)

Symposium Cochairs

Alexei L. Glebov, OptiGrate Corporation (United States)

Klaus P. Streubel, OSRAM GmbH (Germany)

Program Track Chair

James G. Grote, Air Force Research Laboratory (United States)

Conference Chairs

Markus Betz, Technische Universität Dortmund (Germany)

Abdulhakem Y. Elezzabi, University of Alberta (Canada)

Jin-Joo Song, University of California, San Diego (United States)

Kong-Thon Tsen, Arizona State University (United States)

Conference Program Committee

Mischa Bonn, FOM Institute for Atomic and Molecular Physics

(Netherlands)

Yujie J. Ding, Lehigh University (United States)

Jan A. Gaj, University of Warsaw (Poland)

Kazuhiko Hirakawa, The University of Tokyo (Japan)

Rupert Huber, Universität Regensburg (Germany)

Robert A. Kaindl, Lawrence Berkeley National Laboratory (United States)

Dai-Sik Kim, Seoul National University (Korea, Republic of)

Torsten Meier, Universität Paderborn (Germany)

Walter Pfeiffer, Universität Bielefeld (Germany)

Mark I. Stockman, Georgia State University (United States)

Chi-Kuang Sun, National Taiwan University (Taiwan)

Fabrice Vallee, Université Claude Bernard Lyon 1 (France)

Klaas Wynne, University of Glasgow (United Kingdom)

Session Chairs

1 THz Spectroscopy of Water and Biological Molecules I

Abdulhakem Y. Elezzabi, University of Alberta (Canada) 
2 THz Spectroscopy of Water and Biological Molecules II

Klaas Wynne, University of Glasgow (United Kingdom)

3 Coherent Spin Dynamics

Markus Betz, Technische Universität Dortmund (Germany)

$4 \quad$ Ultrafast Phenomena in Semiconductors and Insulators

Stefan Linden, Rheinische Friedrich-Wilhelms- Universitäł Bonn

(Germany)

5 Nonlinear Metamaterials

Javier Aizpurua, Centro de Fisica de Materiales (Spain)

6 Nonlinear $\mathrm{THz}$ Interaction and Spectroscopy

Rupert Huber, Universität Regensburg (Germany)

7 Ultrafast Processes in Graphene and Carbon Nanotubes I

Jin-Joo Song, University of California, San Diego (United States)

Mark S. Sherwin, University of California, Santa Barbara (United States)

8 Ultrafast Nanoplasmonics

Abdulhakem Y. Elezzabi, University of Alberta (Canada)

9 Novel Nanoplasmonic Spectroscopy

Markus Betz, Technische Universität Dortmund (Germany)

10 Strong-Field and Attosecond Phenomena

Abdulhakem Y. Elezzabi, University of Alberta (Canada)

11 Ultrafast Plasmonic Nanoscopy

Georg Herink, Universität Göttingen (Germany)

12 Nonlinear and Coherent Optical Phenomena

Jin-Joo Song, University of California, San Diego (United States)

Markus B. Raschke, University of Colorado at Boulder (United States)

13 Ultrafast Processes in Graphene and Carbon Nanotubes II

Andreas Knorr, Technische Universität Berlin (Germany)

14 Ultrafast Nanophotonics

Markus Lippitz, Max-Planck-Institut für Festkörperforschung (Germany)

15 Plasmonics and Metamaterials

Natalia Del Fatti, Université Claude Bernard Lyon 1 (France) 